\title{
On the convergence of hybrid projection algorithms for total quasi-asymptotically pseudo-contractive mapping
}

Zi-Ming Wang ${ }^{1 *}$ and Yongfu Su

${ }^{*}$ Correspondence:
wangziming@ymail.com
${ }^{1}$ Department of Foundation,
Shandong Yingcai University, Jinan,
250104, P.R. China
Full list of author information is
available at the end of the article

available at the end of the article

\begin{abstract}
In this paper, a hybrid projection algorithm for a total quasi-asymptotically pseudo-contractive mapping is introduced in a Hilbert space. A strong convergence theorem of the proposed algorithm to a fixed point of a total quasi-asymptotically pseudo-contractive mapping is proved. Our main result extends and improves many corresponding results.

MSC: $47 \mathrm{H} 05 ; 47 \mathrm{HO} 0$
\end{abstract}

Keywords: total quasi-asymptotically pseudo-contractive; hybrid projection algorithm; fixed point; Hilbert space

\section{Introduction}

Throughout this paper, we always assume that $H$ is a real Hilbert space, whose inner product and norm are denoted by $\langle\cdot, \cdot\rangle$ and $\|\cdot\|$. The symbol $\rightarrow$ is denoted by a strong convergence. Let $C$ be a nonempty closed and convex subset of $H$, and let $T: C \rightarrow C$ be a mapping. In this paper, we denote the fixed point set of $T$ by $\mathcal{F}(T)$, that is, $\mathcal{F}(T):=\{x \in$ $C: T x=x\}$.

Recall that $T$ is said to be asymptotically nonexpansive if there exists a sequence $\left\{k_{n}\right\} \subset$ $[1, \infty)$ with $k_{n} \rightarrow 1$ as $n \rightarrow \infty$ such that

$$
\left\|T^{n} x-T^{n} y\right\| \leq k_{n}\|x-y\|, \quad \forall n \geq 1, \forall x, y \in C .
$$

The class of asymptotically nonexpansive mappings was introduced by Goebel and Kirk [1] as a generalization of the class of nonexpansive mappings.

$T$ is said to be asymptotically nonexpansive in the intermediate sense if it is continuous and the following inequality holds:

$$
\limsup _{n \rightarrow \infty} \sup _{x, y \in C}\left(\left\|T^{n} x-T^{n} y\right\|-\|x-y\|\right) \leq 0 .
$$

Noticing that if we define

$$
\rho_{n}=\max \left\{0, \sup _{x, y \in C}\left(\left\|T^{n} x-T^{n} y\right\|-\|x-y\|\right)\right\},
$$

\section{Springer}

(0) 2013 Wang and Su; licensee Springer. This is an Open Access article distributed under the terms of the Creative Commons Attribution License (http://creativecommons.org/licenses/by/2.0), which permits unrestricted use, distribution, and reproduction in any medium, provided the original work is properly cited. 
then $\rho_{n} \rightarrow 0$ as $n \rightarrow \infty$. It follows that (1.2) is reduced to

$$
\left\|T^{n} x-T^{n} y\right\| \leq\|x-y\|+\rho_{n}, \quad \forall n \geq 1, \forall x, y \in C .
$$

The class of mappings, which are asymptotically nonexpansive in the intermediate sense, was introduced by Bruck et al. [2] (see also [3]). It is worth mentioning that the class of mappings which are asymptotically nonexpansive in the intermediate sense contains properly the class of asymptotically nonexpansive mappings.

Recall that $T$ is said to be asymptotically pseudocontractive if there exists a sequence $\left\{k_{n}\right\} \subset[1, \infty)$ with $k_{n} \rightarrow 1$ as $n \rightarrow \infty$ such that

$$
\left\langle T^{n} x-T^{n} y, x-y\right\rangle \leq k_{n}\|x-y\|^{2}, \quad \forall x, y \in C .
$$

It is not hard to see that (1.5) is equivalent to

$$
\left\|T^{n} x-T^{n} y\right\|^{2} \leq\left(2 k_{n}-1\right)\|x-y\|^{2}+\left\|x-y-\left(T^{n} x-T^{n} y\right)\right\|^{2}, \quad \forall n \geq 1, x, y \in C .
$$

The class of an asymptotically pseudocontractive mapping was introduced by Schu [4] (see also [5]). In [6], Rhoades gave an example to show that the class of asymptotically pseudocontractive mappings contains properly the class of asymptotically nonexpansive mappings, see [6] for more details. Zhou [7] showed that every uniformly Lipschitz and asymptotically pseudocontractive mapping, which is also uniformly asymptotically regular, has a fixed point.

$T$ is said to be an asymptotically pseudocontractive mapping in the intermediate sense if there exists a sequence $\left\{k_{n}\right\} \subset[1, \infty)$ with $k_{n} \rightarrow 1$ as $n \rightarrow \infty$ such that

$$
\limsup _{n \rightarrow \infty} \sup _{x, y \in C}\left(\left\langle T^{n} x-T^{n} y, x-y\right\rangle-k_{n}\|x-y\|^{2}\right) \leq 0 .
$$

Put

$$
\tau_{n}=\max \left\{0, \sup _{x, y \in C}\left(\left\langle T^{n} x-T^{n} y, x-y\right\rangle-k_{n}\|x-y\|^{2}\right)\right\} .
$$

It follows that $\tau_{n} \rightarrow 0$ as $n \rightarrow \infty$. Then, (1.8) is reduced to the following:

$$
\left\langle T^{n} x-T^{n} y, x-y\right\rangle \leq k_{n}\|x-y\|^{2}+\tau_{n}, \quad \forall n \geq 1, x, y \in C .
$$

The class of asymptotically pseudocontractive mappings in the intermediate sense was introduced by Qin et al. [8].

Recall that $T$ is said to be total asymptotically pseudocontractive if there exist sequences $\left\{k_{n}\right\},\left\{v_{n}\right\} \subset[0, \infty)$ with $k_{n}, v_{n} \rightarrow 0$ as $n \rightarrow \infty$ such that

$$
\left\langle T^{n} x-T^{n} y, x-y\right\rangle \leq\|x-y\|^{2}+k_{n} \phi(\|x-y\|)+v_{n}, \quad \forall n \geq 1, x, y \in C,
$$

where $\phi:[0, \infty) \rightarrow[0, \infty)$ is a continuous and strictly increasing function with $\phi(0)=0$. The class of a total asymptotically pseudocontractive mapping was introduced by Qin [9]. 
It is easy to see that (1.10) is equivalent to the following: for all $n \geq 1, x, y \in C$,

$$
\left\|T^{n} x-T^{n} y\right\|^{2} \leq\|x-y\|^{2}+2 k_{n} \phi(\|x-y\|)+\left\|x-y-\left(T^{n} x-T^{n} y\right)\right\|^{2}+2 v_{n} .
$$

If $\phi(\lambda)=\lambda^{2}$, then (1.10) is reduced to

$$
\left\langle T^{n} x-T^{n} y, x-y\right\rangle \leq\left(1+k_{n}\right)\|x-y\|^{2}+v_{n}, \quad \forall n \geq 1, x, y \in C .
$$

Put

$$
v_{n}=\max \left\{0, \sup _{x, y \in C}\left(\left\langle T^{n} x-T^{n} y, x-y\right\rangle-\left(1+k_{n}\right)\|x-y\|^{2}\right)\right\} .
$$

If $\phi(\lambda)=\lambda^{2}$, then the class of total asymptotically pseudocontractive mappings is reduced to the class of asymptotically pseudocontractive mappings in the intermediate sense.

In this paper, we introduce and study the following mapping.

Definition 1.1 A mapping $T: C \rightarrow C$ is said to be total quasi-asymptotically pseudocontractive if $\mathcal{F}(T) \neq \emptyset$, and there exist sequences $\left\{\mu_{n}\right\} \subset[0, \infty)$ and $\left\{\xi_{n}\right\} \subset[0, \infty)$ with $\mu_{n} \rightarrow 0$ and $\xi_{n} \rightarrow 0$ as $n \rightarrow \infty$ such that

$$
\left\langle T^{n} x-p, x-p\right\rangle \leq\|x-p\|^{2}+\mu_{n} \phi(\|x-p\|)+\xi_{n}, \quad \forall n \geq 1, x \in C, p \in \mathcal{F}(T)
$$

where $\phi:[0, \infty) \rightarrow[0, \infty)$ is a continuous and strictly increasing function with $\phi(0)=0$.

It is easy to see that (1.14) is equivalent to the following:

$$
\begin{aligned}
\left\|T^{n} x-p\right\|^{2} \leq & \|x-p\|^{2}+2 \mu_{n} \phi(\|x-p\|) \\
& +\left\|x-T^{n} x\right\|^{2}+2 \xi_{n}, \quad \forall n \geq 1, x \in C, p \in \mathcal{F}(T) .
\end{aligned}
$$

Remark 1 It is clear that every total asymptotically pseudo-contractive mapping with $F(T) \neq \varnothing$ is total quasi-asymptotically pseudo-contractive, but the converse maybe not true.

Remark 2 If $\phi(\lambda)=\lambda^{2}$, the (1.14) is reduced to

$$
\left\langle T^{n} x-p, x-p\right\rangle \leq\left(1+\mu_{n}\right)\|x-p\|^{2}+\xi_{n}, \quad \forall n \geq 1, x \in C, p \in \mathcal{F}(T) .
$$

Remark 3 Put

$$
\xi_{n}=\max \left\{0, \sup _{x, y \in C}\left(\left\langle T^{n} x-p, x-p\right\rangle-\left(1+\mu_{n}\right)\|x-p\|^{2}\right)\right\} .
$$

If $\phi(\lambda)=\lambda^{2}$, then the class of total quasi-asymptotically pseudo-contractive mappings is reduced to the class of quasi-asymptotically pseudo-contractive mappings in the intermediate sense. 
Recently, the iterative approximation of fixed points for asymptotically pseudo-contractive mappings, total asymptotically pseudo-contractive mappings in Hilbert, or Banach spaces has been studied extensively by many authors, see, for example, [7, 9-13]. In this paper, we shall consider and study a total quasi-asymptotically pseudo-contractive mapping as a generalization of (total) asymptotically pseudo-contractive mappings. Furthermore, we shall introduce an iterative algorithm for finding a fixed point of a total quasiasymptotically pseudo-contractive mapping.

\section{Preliminaries}

A mapping $T: C \rightarrow C$ is said to be uniformly $L$-Lipschitzian if there exists some $L>0$ such that

$$
\left\|T^{n} x-T^{n} y\right\| \leq L\|x-y\|, \quad \forall x, y \in C, n \geq 1 .
$$

Let $C$ be a nonempty closed convex subset of a real Hilbert space $H$. For every point $x \in$ $H$, there exists a unique nearest point in $C$, denoted by $P_{C} x$, such that $\left\|x-P_{C} x\right\| \leq\|x-y\|$ holds for all $y \in C$, where $P_{C}$ is said to be the metric projection of $H$ onto $C$.

In order to prove our main results, we also need the following lemmas.

Lemma 2.1 [14] Let $C$ be a nonempty closed convex subset of a real Hilbert space $H$ and let $P_{C}$ be the metric projection from $H$ onto $C$ (i.e., for $x \in H, P_{C}$ is the only point in $C$ such that $\left.\left\|x-P_{C} x\right\|=\inf \{\|x-z\|: z \in C\}\right)$. Given $x \in H$ and $z \in C$, then $z=P_{C} x$ if and only if the relation

$$
\langle x-z, y-z\rangle \leq 0, \quad \forall y \in C
$$

holds.

Lemma 2.2 Let $C$ be a nonempty bounded and closed convex subset of a real Hilbert space $H$. Let $T: C \rightarrow C$ be a uniformly L-Lipschitzian and total quasi-asymptotically pseudo-contractive mapping with $\mathcal{F}(T) \neq \emptyset$. Suppose there exist positive constants $M$ and $M^{*}$ such that $\phi(\zeta) \leq M^{*} \zeta^{2}$ for all $\zeta>M$. Then $\mathcal{F}(T)$ is a closed convex subset of $C$.

Proof Since $\phi$ is an increasing function, it follows that $\phi(\zeta) \leq \phi(M)$ if $\zeta \leq M$ and $\phi(\zeta) \leq$ $M^{*} \zeta^{2}$ if $\zeta \geq M$. In either case, we can always obtain that

$$
\phi(\zeta) \leq \phi(M)+M^{*} \zeta^{2}
$$

Since $T$ is uniformly $L$-Lipschitzian continuous, $\mathcal{F}(T)$ is closed. We need to show that $\mathcal{F}(T)$ is convex. To this end, let $p_{i} \in \mathcal{F}(T)(i=1,2)$, and write $p=t p_{1}+(1-t) p_{2}$ for $t \in(0,1)$. We take $\alpha \in\left(0, \frac{1}{1+L}\right)$, and define $y_{\alpha, n}=(1-\alpha) p+\alpha T^{n} p$ for each $n \in \mathbb{N}$. Then, for all $z \in \mathcal{F}(T)$, we have from (2.3) that

$$
\begin{aligned}
\left\|p-T^{n} p\right\|^{2} & =\left\langle p-T^{n} p, p-T^{n} p\right\rangle \\
& =\frac{1}{\alpha}\left\langle p-y_{\alpha, n}, p-T^{n} p\right\rangle \\
& =\frac{1}{\alpha}\left\langle p-y_{\alpha, n}, p-T^{n} p-\left(y_{\alpha, n}-T^{n} y_{\alpha, n}\right)\right\rangle
\end{aligned}
$$




$$
\begin{aligned}
& +\frac{1}{\alpha}\left\langle p-y_{\alpha, n}, y_{\alpha, n}-T^{n} y_{\alpha, n}\right\rangle \\
\leq & \frac{1+L}{\alpha}\left\|p-y_{\alpha, n}\right\|^{2}+\frac{1}{\alpha}\left\langle p-z, y_{\alpha, n}-T^{n} y_{\alpha, n}\right\rangle \\
& +\frac{1}{\alpha}\left\langle z-y_{\alpha, n}, y_{\alpha, n}-T^{n} y_{\alpha, n}\right\rangle \\
= & \frac{1+L}{\alpha}\left\|p-y_{\alpha, n}\right\|^{2}+\frac{1}{\alpha}\left\langle p-z, y_{\alpha, n}-T^{n} y_{\alpha, n}\right\rangle \\
& +\frac{1}{\alpha}\left\langle z-y_{\alpha, n}, y_{\alpha, n}-z+z-T^{n} y_{\alpha, n}\right\rangle \\
\leq & \frac{1+L}{\alpha}\left\|p-y_{\alpha, n}\right\|^{2}+\frac{1}{\alpha}\left\langle p-z, y_{\alpha, n}-T^{n} y_{\alpha, n}\right\rangle \\
& +\frac{1}{\alpha}\left\{\mu_{n}\left[\phi(M)+M^{*}(\operatorname{diam} C)^{2}\right]+\xi_{n}\right\} \\
= & \alpha(1+L)\left\|p-T^{n} p\right\|^{2}+\frac{1}{\alpha}\left\langle p-z, y_{\alpha, n}-T^{n} y_{\alpha, n}\right\rangle \\
& +\frac{1}{\alpha}\left\{\mu_{n}\left[\phi(M)+M^{*}(\operatorname{diam} C)^{2}\right]+\xi_{n}\right\} .
\end{aligned}
$$

This implies that

$$
\begin{aligned}
\alpha[1-\alpha(1+L)]\left\|p-T^{n} p\right\|^{2} \leq & \left\langle p-z, y_{\alpha, n}-T^{n} y_{\alpha, n}\right\rangle \\
& +\mu_{n}\left[\phi(M)+M^{*}(\operatorname{diam} C)^{2}\right]+\xi_{n} .
\end{aligned}
$$

Now, we take $z=p_{i}(i=1,2)$ in $(2.4)$, multiplying $t$ and $(1-t)$ on the both sides of the above inequality (2.4), respectively, and adding up, and we can get

$$
\alpha[1-\alpha(1+L)]\left\|p-T^{n} p\right\|^{2} \leq \mu_{n}\left[\phi(M)+M^{*}(\operatorname{diam} C)^{2}\right]+\xi_{n}
$$

Letting $n \rightarrow \infty$ in (2.5), we obtain $T^{n} p \rightarrow p$. Since $T$ is continuous, we have $T^{n+1} p \rightarrow T p$ as $n \rightarrow \infty$, therefore, $p=T p$. This proves that $\mathcal{F}(T)$ is a closed convex subset of $C$.

\section{Main results}

In this section, we shall give our main results of this paper.

Theorem 3.1 Let $C$ be a nonempty bounded and closed convex subset of a real Hilbert space $H$. Let $T: C \rightarrow C$ be a uniformly L-Lipschitzian and total quasi-asymptotically pseudo-contractive mapping with $\mathcal{F}(T) \neq \emptyset$. Suppose that there exist positive constants $M$ and $M^{*}$ such that $\phi(\zeta) \leq M^{*} \zeta^{2}$ for all $\zeta>M$. Let $\left\{x_{n}\right\}$ be a sequence generated by the following iterative scheme:

$$
\left\{\begin{array}{l}
x_{1} \in C \quad \text { chosen arbitrarily, } \\
C_{1}=C, \quad Q_{1}=C, \\
y_{n}=\left(1-\alpha_{n}\right) x_{n}+\alpha_{n} T^{n} x_{n}, \\
C_{n+1}=\left\{z \in C_{n}: \alpha_{n}\left[1-\alpha_{n}(1+L)\right]\left\|x_{n}-T^{n} x_{n}\right\|^{2} \leq\left\langle x_{n}-z, y_{n}-T^{n} y_{n}\right\rangle+\theta_{n}\right\}, \\
Q_{n+1}=\left\{z \in Q_{n}:\left\langle x_{n}-z, x_{1}-x_{n}\right\rangle \geq 0\right\}, \\
x_{n+1}=P_{C_{n+1} \cap Q_{n+1}} x_{1}, \quad \forall n \geq 1,
\end{array}\right.
$$


where $\theta_{n}=\mu_{n}\left[\phi(M)+M^{*}(\operatorname{diam} C)^{2}\right]+\xi_{n},\left\{\alpha_{n}\right\}$ is a sequence in $[a, b]$ with $a, b \in\left(0, \frac{1}{1+L}\right)$. Then the sequence $\left\{x_{n}\right\}$ converges strongly to a point $P_{\mathcal{F}(T)} x_{1}$, where $P_{\mathcal{F}(T)}$ is the projection from $C$ onto $\mathcal{F}(T)$.

Proof We split the proof into seven steps.

Step 1 . Show that $P_{\mathcal{F}(T)} x_{1}$ is well defined for every $x_{1} \in C$.

By Lemma 2.2, we know that $\mathcal{F}(T)$ is a closed and convex subset of $C$. Therefore, in view of the assumption of $\mathcal{F}(T) \neq \emptyset, P_{\mathcal{F}(T)} x_{1}$ is well defined for every $x_{1} \in C$.

Step 2. Show that $C_{n}$ and $Q_{n}$ are closed and convex for all $n \geq 1$.

From the definitions of $C_{n}$ and $Q_{n}$, it is obvious that $C_{n}$ and $Q_{n}$ are closed and convex for all $n \geq 1$. We omit the details.

Step 3. Show that $\mathcal{F}(T) \subset C_{n} \cap Q_{n}$ for all $n \geq 1$.

To this end, we first prove that $\mathcal{F}(T) \subset C_{n}$ for all $n \geq 1$. This can be proved by induction on $n$. It is obvious that $\mathcal{F}(T) \subset C_{1}=C$. Assume that $\mathcal{F}(T) \subset C_{n}$ for some $n \in \mathbb{N}$. Then, using the uniform $L$-Lipschitzian continuity of $T$, the total quasi-asymptotic pseudocontractiveness of $T$ and (2.3), we have for any $w \in \mathcal{F}(T)$ that

$$
\begin{aligned}
\left\|x_{n}-T^{n} x_{n}\right\|^{2}= & \left\langle x_{n}-T^{n} x_{n}, x_{n}-T^{n} x_{n}\right\rangle \\
= & \frac{1}{\alpha_{n}}\left\langle x_{n}-y_{n}, x_{n}-T^{n} x_{n}\right\rangle \\
= & \frac{1}{\alpha_{n}}\left\langle x_{n}-y_{n}, x_{n}-T^{n} x_{n}-\left(y_{n}-T^{n} y_{n}\right)\right\rangle+\frac{1}{\alpha_{n}}\left\langle x_{n}-y_{n}, y_{n}-T^{n} y_{n}\right\rangle \\
= & \frac{1}{\alpha_{n}}\left\langle x_{n}-y_{n}, x_{n}-T^{n} x_{n}-\left(y_{n}-T^{n} y_{n}\right)\right\rangle \\
& +\frac{1}{\alpha_{n}}\left\langle x_{n}-w+w-y_{n}, y_{n}-T^{n} y_{n}\right\rangle \\
\leq & \frac{1+L}{\alpha_{n}}\left\|x_{n}-y_{n}\right\|^{2}+\frac{1}{\alpha_{n}}\left\langle x_{n}-w, y_{n}-T^{n} y_{n}\right\rangle \\
& +\frac{1}{\alpha_{n}}\left\langle w-y_{n}, y_{n}-T^{n} y_{n}\right\rangle \\
= & \frac{1+L}{\alpha_{n}}\left\|x_{n}-y_{n}\right\|^{2}+\frac{1}{\alpha_{n}}\left\langle x_{n}-w, y_{n}-T^{n} y_{n}\right\rangle \\
& +\frac{1}{\alpha_{n}}\left\langle w-y_{n}, y_{n}-w+w-T^{n} y_{n}\right\rangle \\
= & \frac{1+L}{\alpha_{n}}\left\|x_{n}-y_{n}\right\|^{2}+\frac{1}{\alpha_{n}}\left\langle x_{n}-w, y_{n}-T^{n} y_{n}\right\rangle \\
& -\frac{1}{\alpha_{n}}\left\|w-y_{n}\right\|^{2}+\frac{1}{\alpha_{n}}\left\langle w-y_{n}, w-T^{n} y_{n}\right\rangle \\
\leq & \frac{1+L}{\alpha_{n}}\left\|x_{n}-y_{n}\right\|^{2}+\frac{1}{\alpha_{n}}\left\langle x_{n}-w, y_{n}-T^{n} y_{n}\right\rangle \\
& +\frac{1}{\alpha_{n}}\left\{\mu_{n}\left[\phi(M)+M^{*}(\operatorname{diam} C)^{2}\right]+\xi_{n}\right\} \\
= & (1+L) \alpha_{n}\left\|x_{n}-T^{n} x_{n}\right\|^{2}+\frac{1}{\alpha_{n}}\left\langle x_{n}-w, y_{n}-T^{n} y_{n}\right\rangle \\
& +\frac{1}{\alpha_{n}}\left\{\mu_{n}\left[\phi(M)+M^{*}(\mathrm{diam} C)^{2}\right]+\xi_{n}\right\}, \\
& \\
&
\end{aligned}
$$


which implies that

$$
\alpha_{n}\left[1-\alpha_{n}(1+L)\right]\left\|x_{n}-T^{n} x_{n}\right\|^{2} \leq\left\langle x_{n}-w, y_{n}-T^{n} y_{n}\right\rangle+\mu_{n}\left[\phi(M)+M^{*}(\operatorname{diam} C)^{2}\right]+\xi_{n},
$$

which shows that $w \in C_{n+1}$. By the mathematical induction principle, $\mathcal{F}(T) \subset C_{n}$ for all $n \geq 1$.

Next, we prove $\mathcal{F}(T) \subset Q_{n}$ for all $n \geq 1$. By induction, for $n=1$, we have $\mathcal{F}(T) \subset C=Q_{1}$. Assume that $\mathcal{F}(T) \subset Q_{n}$ for some $n \in \mathbb{N}$. Since $x_{n}$ is the projection of $x_{1}$ onto $C_{n} \cap Q_{n}$, by Lemma 2.1, we have

$$
\left\langle x_{n}-z, x_{1}-x_{n}\right\rangle \geq 0, \quad \forall z \in C_{n} \cap Q_{n}
$$

Since $\mathcal{F}(T) \subset C_{n} \cap Q_{n}$, we easily see that

$$
\left\langle x_{n}-w, x_{1}-x_{n}\right\rangle \geq 0, \quad \forall w \in \mathcal{F}(T)
$$

which implies that $\mathcal{F}(T) \subset Q_{n+1}$. This proves that $\mathcal{F}(T) \subset C_{n} \cap Q_{n}$ for all $n \geq 1$.

Step 4. Show that $\lim _{n \rightarrow \infty}\left\|x_{n}-x_{1}\right\|$ exists.

In view of (3.1) and Lemma 2.1, we have $x_{n}=P_{Q_{n}} x_{1}$ and $x_{n+1} \in Q_{n}$, which implies

$$
\left\|x_{n}-x_{1}\right\| \leq\left\|x_{n+1}-x_{1}\right\|, \quad \forall n \geq 1
$$

On the other hand, since $\mathcal{F}(T) \subset Q_{n}$, we also have

$$
\left\|x_{n}-x_{1}\right\| \leq\left\|w-x_{1}\right\|, \quad \forall w \in \mathcal{F}(T), \forall n \geq 1 .
$$

Therefore, $\lim _{n \rightarrow \infty}\left\|x_{n}-x_{1}\right\|$ exists and $\left\{x_{n}\right\}$ is bounded.

Step 5 . Show that $\left\{x_{n}\right\}$ is a Cauchy sequence.

Noticing the construction of $C_{n}$, one has $C_{m} \subset C_{n}$ and $x_{m}=P_{C_{m}} x_{1} \in C_{n}$ for any positive integer $m>n$. From (3.2), we have

$$
\left\langle x_{n}-x_{n+m}, x_{1}-x_{n}\right\rangle \geq 0 \text {. }
$$

It follows that

$$
\begin{aligned}
\left\|x_{n}-x_{n+m}\right\|^{2} & =\left\|x_{n}-x_{1}+x_{1}-x_{n+m}\right\|^{2} \\
& =\left\|x_{n}-x_{1}\right\|^{2}+\left\|x_{1}-x_{n+m}\right\|^{2}-2\left\langle x_{1}-x_{n}, x_{1}-x_{n+m}\right\rangle \\
& =\left\|x_{n}-x_{1}\right\|^{2}+\left\|x_{1}-x_{n+m}\right\|^{2}-2\left\langle x_{1}-x_{n}, x_{1}-x_{n}+x_{n}-x_{n+m}\right\rangle \\
& \leq\left\|x_{1}-x_{n+m}\right\|^{2}-\left\|x_{n}-x_{1}\right\|^{2}-\left\langle x_{1}-x_{n}, x_{n}-x_{n+m}\right\rangle \\
& \leq\left\|x_{1}-x_{n+m}\right\|^{2}-\left\|x_{n}-x_{1}\right\|^{2} .
\end{aligned}
$$

Letting $n \rightarrow \infty$ in (3.4), one has $\lim _{n \rightarrow \infty}\left\|x_{n}-x_{n+m}\right\|=0, \forall m \geq n$. Hence, $\left\{x_{n}\right\}$ is a Cauchy sequence. Since $H$ is a Hilbert space and $C$ is closed and convex, one can assume that $x_{n} \rightarrow q \in C$ as $n \rightarrow \infty$.

Step 6. Show that $\lim _{n \rightarrow \infty}\left\|x_{n}-T x_{n}\right\|=0$. 
It follows from $x_{n+1} \in C_{n}$ and (3.1) that

$$
\begin{aligned}
\alpha_{n}\left[1-\alpha_{n}(1+L)\right]\left\|x_{n}-T^{n} x_{n}\right\|^{2} & \leq\left\langle x_{n}-x_{n+1}, y_{n}-T^{n} y_{n}\right\rangle+\theta_{n} \\
& \leq\left\|x_{n}-x_{n+1}\right\|\left\|y_{n}-T^{n} y_{n}\right\|+\theta_{n} .
\end{aligned}
$$

Since $\left\{y_{n}\right\}$ is bounded, $\left\{T^{n} y_{n}\right\}$ is bounded, $\lim _{n \rightarrow \infty}\left\|x_{n+1}-x_{n}\right\|=0$ and $\alpha_{n} \in(a, b)$, we have from (3.5) that

$$
\lim _{n \rightarrow \infty}\left\|x_{n}-T^{n} x_{n}\right\|=0
$$

On the other hand, we notice that

$$
\begin{aligned}
\left\|x_{n}-T x_{n}\right\| \leq & \left\|x_{n}-x_{n+1}\right\|+\left\|x_{n+1}-T^{n+1} x_{n+1}\right\| \\
& +\left\|T^{n+1} x_{n+1}-T^{n+1} x_{n}\right\|+\left\|T^{n+1} x_{n}-T x_{n}\right\| \\
\leq & (1+L)\left\|x_{n}-x_{n+1}\right\|+\left\|x_{n+1}-T^{n+1} x_{n+1}\right\|+L\left\|T^{n} x_{n}-x_{n}\right\| .
\end{aligned}
$$

From $\lim _{n \rightarrow \infty}\left\|x_{n+1}-x_{n}\right\|=0$ and $\lim _{n \rightarrow \infty}\left\|x_{n}-T^{n} x_{n}\right\|=0$, we have

$$
\lim _{n \rightarrow \infty}\left\|x_{n}-T x_{n}\right\|=0
$$

It follows that $T x_{n} \rightarrow q$ as $n \rightarrow \infty$. Since $\mathrm{T}$ is continuous, one has that $q$ is a fixed point of $T$; that is, $q \in \mathcal{F}(T)$.

Step 7. Finally, we prove $q=P_{\mathcal{F}(T)} x_{1}$.

By taking the limit in (3.3), we have

$$
\left\langle q-w, x_{1}-q\right\rangle \geq 0, \quad \forall w \in \mathcal{F}(T),
$$

which implies that $q=P_{\mathcal{F}(T)} x_{1}$ by using Lemma 2.1. This completes the proof.

Since every total asymptotically pseudo-contractive mapping with $\mathcal{F}(T) \neq \emptyset$ is total quasi-asymptotically pseudo-contractive, we immediately obtain the following corollary:

Corollary 3.2 Let $C$ be a nonempty bounded and closed convex subset of a real Hilbert space H. Let $T: C \rightarrow C$ be a uniformly L-Lipschitzian and total asymptotically pseudocontractive mapping with $\mathcal{F}(T) \neq \emptyset$. Suppose there exist positive constants $M$ and $M^{*}$ such that $\phi(\zeta) \leq M^{*} \zeta^{2}$ for all $\zeta>M$. Let $\left\{x_{n}\right\}$ be a sequence generated by the following iterative scheme:

$$
\left\{\begin{array}{l}
x_{1} \in C \quad \text { chosen arbitrarily, } \\
C_{1}=C, \quad Q_{1}=C, \\
y_{n}=\left(1-\alpha_{n}\right) x_{n}+\alpha_{n} T^{n} x_{n}, \\
C_{n+1}=\left\{z \in C_{n}: \alpha_{n}\left[1-\alpha_{n}(1+L)\right]\left\|x_{n}-T^{n} x_{n}\right\|^{2} \leq\left\langle x_{n}-z, y_{n}-T^{n} y_{n}\right\rangle+\theta_{n}\right\}, \\
Q_{n+1}=\left\{z \in Q_{n}:\left\langle x_{n}-z, x_{1}-x_{n}\right\rangle \geq 0\right\}, \\
x_{n+1}=P_{C_{n+1} \cap Q_{n+1}} x_{1}, \quad \forall n \geq 1,
\end{array}\right.
$$


where $\theta_{n}=\mu_{n}\left[\phi(M)+M^{*}(\operatorname{diam} C)^{2}\right]+\xi_{n},\left\{\alpha_{n}\right\}$ is a sequence in $[a, b]$ with $a, b \in\left(0, \frac{1}{1+L}\right)$. Then the sequence $\left\{x_{n}\right\}$ converges strongly to a point $P_{\mathcal{F}(T)} x_{1}$, where $P_{\mathcal{F}(T)}$ is the projection from $C$ onto $\mathcal{F}(T)$.

Remark 3.3 Since the class of the total quasi-asymptotically pseudo-contractive mappings includes the class of asymptotically pseudocontractive mappings, the class of asymptotically pseudocontractive mappings in the intermediate sense, the class of the total asymptotically pseudo-contractive mappings, the class of quasi-asymptotically pseudocontractive mappings in the intermediate sense as special cases, Theorem 3.1 improves the corresponding results in Zhou [7], Qin et al. [9], Chang [10] and Qin et al. [12].

Competing interests

The authors declare that they have no competing interests.

Authors' contributions

All authors read and approved the final manuscript.

\section{Author details}

'Department of Foundation, Shandong Yingcai University, Jinan, 250104, P.R. China. ${ }^{2}$ Department of Mathematics, Tianjin Polytechnic University, Tianjin, 300387, P.R. China

\section{Acknowledgements}

The authors are grateful to the referees for their helpful and useful comments. The first author is supported by the Project of Shandong Province Higher Educational Science and Technology Program (grant No. J13LI51) and the Natural Science Foundation of Shandong Yingcai University (grant No. 12YCZDZR03). The second author is supported by the National Natural Science Foundation of China under grant (11071279) and the Project of Shandong Province Higher Educational Science and Technology Program (grant No. J13LI51).

Received: 25 April 2013 Accepted: 25 July 2013 Published: 9 August 2013

\section{References}

1. Goebel, K, Kirk, WA: A fixed point theorem for asymptotically nonexpansive mappings. Proc. Am. Math. Soc. 35, 171-174 (1972)

2. Bruck, R, Kuczumow, T, Reich, S: Convergence of iterates of asymptotically nonexpansive mappings in Banach spaces with the uniform Opial property. Colloq. Math. 65, 169-179 (1993)

3. Kirk, WA: Fixed point theorems for non-Lipschitzian mappings of asymptotically nonexpansive type. Isr. J. Math. 17, 339-346 (1974)

4. Schu, J: Iterative construction of fixed points of asymptotically nonexpansive mappings. J. Math. Anal. Appl. 158, 407-413 (1991)

5. Schu, J: Weak and strong convergence to fixed points of asymptotically nonexpansive mappings. Bull. Aust. Math. Soc. 43, 153-159 (1991)

6. Rhoades, BE: Comments on two fixed point iteration methods. J. Math. Anal. Appl. 56, 741-750 (1976)

7. Zhou, $\mathrm{H}$ : Demiclosedness principle with applications for asymptotically pseudo-contractions in Hilbert spaces. Nonlinear Anal., Theory Methods Appl. 70, 3140-3145 (2009)

8. Qin, X, Cho, SY, Kim, JK: Convergence theorems on asymptotically pseudocontractive mappings in the intermediate sense. Fixed Point Theory Appl. 2010, Article ID 186874 (2010)

9. Qin, X, Cho, SY, Kang, SM: A weak convergence theorem for total asymptotically pseudocontractive mappings in Hilbert spaces. Fixed Point Theory Appl. 2011, Article ID 859795 (2011)

10. Chang, SS: Some results for asymptotically pseudo-contractive mappings and asymptotically nonexpansive mappings. Proc. Am. Math. Soc. 129, 845-853 (2001)

11. Osilike, MO, Akuchu, BG: Common fixed points of a finite family of asymptotically pseudocontractive maps. Fixed Point Theory Appl. 2, 81-88 (2004)

12. Qin, X, Kim, JK, Wang, T: On the convergence of implicit iterative processes for asymptotically pseudocontractive mappings in the intermediate sense. Abstr. Appl. Anal. 2011, Article ID 468716 (2011)

13. Ding, C, Quan, J: A strong convergence theorem for total asymptotically pseudocontractive mappings in Hilbert spaces. Abstr. Appl. Anal. 2012, Article ID 127851 (2012)

14. Zhou, H: Strong convergence theorems for a family of Lipschitz quasi-pseudo-contractions in Hilbert spaces. Nonlinear Anal. 71, 120-125 (2009)

doi:10.1186/1029-242X-2013-375

Cite this article as: Wang and Su: On the convergence of hybrid projection algorithms for total quasi-asymptotically pseudo-contractive mapping. Journal of Inequalities and Applications 2013 2013:375. 